\title{
Meta-analysis of haematoma volume, haematoma expansion and mortality in intracerebral haemorrhage associated with oral anticoagulant use
}

\author{
David J. Seiffge \\ Stefan T. Engelter ${ }^{2,7} \cdot$ David J. Werring ${ }^{1}$
}

Received: 5 July 2019 / Revised: 5 September 2019 / Accepted: 10 September 2019 / Published online: 20 September 2019

(c) The Author(s) 2019

\begin{abstract}
Objective To obtain precise estimates of age, haematoma volume, secondary haematoma expansion (HE) and mortality for patients with intracerebral haemorrhage (ICH) taking oral anticoagulants [Vitamin K antagonists (VKA-ICH) or nonVitamin $\mathrm{K}$ antagonist oral anticoagulants (NOAC-ICH)] and those not taking oral anticoagulants (non-OAC ICH) at ICH symptom onset.

Methods We conducted a systematic review and meta-analysis of studies comparing VKA-ICH or NOAC-ICH or both with non-OAC ICH. Primary outcomes were haematoma volume (in ml), HE, and mortality (in-hospital and 3-month). We calculated odds ratios (ORs) using the Mantel-Haenszel random-effects method and corresponding 95\% confidence intervals (95\% CI) and determined the mean ICH volume difference.

Results We identified 19 studies including data from 16,546 patients with VKA-ICH and 128,561 patients with non-OAC ICH. Only 2 studies reported data on 4943 patients with NOAC-ICH. Patients with VKA-ICH were significantly older than patients with non-OAC ICH (mean age difference: 5.55 years, 95\% CI 4.03-7.07, $p<0.0001, I^{2}=92 \%, p<0.001$ ). Haematoma volume was significantly larger in VKA-ICH with a mean difference of $9.66 \mathrm{ml}(95 \% \mathrm{CI} 6.24-13.07 \mathrm{ml}, p<0.00001$; $\left.I^{2}=42 \%, p=0.05\right)$. HE occurred significantly more often in VKA-ICH (OR 2.96, 95\%CI 1.74-4.97, $\left.p<0.00001 ; I^{2}=65 \%\right)$. VKA-ICH was associated with significantly higher in-hospital mortality (VKA-ICH: $32.8 \%$ vs. non-OAC ICH: 22.4\%; OR $1.83,95 \%$ CI 1.61-2.07, $p<0.00001, I^{2}=20 \%, p=0.27$ ) and 3-month mortality (VKA-ICH: $47.1 \%$ vs. non-OAC ICH: $25.5 \%$; OR $2.24,95 \%$ CI $1.52-3.31, p<0.00001, I^{2}=71 \%, p=0.001$ ). We did not find sufficient data for a meta-analysis comparing NOAC-ICH and non-OAC-ICH.

Conclusion This meta-analysis confirms, refines and expands findings from prior studies. We provide precise estimates of key prognostic factors and outcomes for VKA-ICH, which has larger haematoma volume, increased rate of HE and higher mortality compared to non-OAC ICH. There are insufficient data on NOACs.
\end{abstract}

Keywords Oral anticoagulants $\cdot$ Intracerebral haemorrhage $\cdot$ Haematoma volume $\cdot$ Mortality $\cdot$ Haematoma expansion

David J. Seiffge and Martina B. Goeldlin are equally contributing first authors.

Electronic supplementary material The online version of this article (https://doi.org/10.1007/s00415-019-09536-1) contains supplementary material, which is available to authorized users.

David J. Seiffge

david.seiffge@insel.ch

$\triangle$ David J. Werring

d.werring@ucl.ac.uk

Extended author information available on the last page of the article

\section{Background}

Intracerebral haemorrhage (ICH) is a devastating form of stroke with high mortality and morbidity [1]. Oral anticoagulants (OAC) are beneficial in the prevention of ischaemic stroke and systemic embolism in patients with atrial fibrillation. Vitamin K antagonists (VKA) were the only option for OAC therapy for many years, but since 2010, direct thrombin inhibitors [2] (dabigatran) and factor Xa inhibitors [3] (apixaban, edoxaban and rivaroxaban) - termed non-vitamin $\mathrm{K}$ antagonist oral anticoagulants (NOAC) - have emerged as an alternative option. 
Current knowledge about the influence of OAC on haematoma volume, secondary haematoma expansion (HE) and mortality in ICH is mainly based on data from small single centre studies conducted in the 1990s [4, 5] or early 2000s [6-8]. These studies found that ICH in patients on VKA therapy (VKA-ICH) was associated with larger haematoma volume and higher rates of $\mathrm{HE}$ and mortality compared to patients not taking OAC (non-OAC ICH). By contrast, more recent studies were less consistent regarding the influence of VKA: some did not find differences in ICH volume [7, 9, 10], HE [9, 10] or mortality [9]; others found that VKA influences haematoma volume in non-lobar but not lobar ICH location [11], or if the INR was supratherapeutic (>3.0) [12].

Since the introduction of NOACs, several single-center $[13,14]$ and multi-center [15-17] studies analysed differences between VKA-ICH and ICH in patients on NOAC therapy (NOAC-ICH) with heterogeneous results.

We conducted a systematic review and meta-analysis of published studies to determine the most precise available estimates of age, haematoma volume, risk of $\mathrm{HE}$ and mortality for OAC-ICH (VKA-ICH or NOAC-ICH) compared to non-OAC ICH.

\section{Methods}

The report was prepared with respect to the PRISMA recommendations [18]. The analysis was performed in $11 / 2018-01 / 2019$ according to a pre-planned protocol developed by all investigators in 10/2018 (not published).

\section{Search strategy and inclusion/exclusion criteria}

Two investigators (DJS and MBG) independently searched pubmed.gov/MEDLINE for relevant publications on 31 October 2018 and on 14 November 2018. We used the following search terms: [(oral anticoagulants OR Vitamin K antagonists or novel oral anticoagulants or direct oral anticoagulants or non-vitamin $\mathrm{K}$ antagonist oral anticoagulants) and (ICH) AND (mortality or volume)] including different spellings and common abbreviations (i.e. VKA, NOAC, DOAC, ICH).

We applied the following inclusion criteria:

- Original studies published in English,

- Comparing OAC-ICH (either VKA-ICH or NOAC-ICH or both) with non-OAC ICH.

- Recruiting patients from the same population (hospitalbased or population-based studies).

- Reporting at least one of the following outcomes for both types of ICH (OAC-ICH and non-OAC ICH): mortality, haematoma volume or HE.
We applied the following exclusion criteria:

- Study with less than 10 participants with OAC-ICH.

- Studies reporting Matched cohorts rather than consecutive patients.

\section{Data collection}

Two author (DJS and MBG) independently performed the literature research and screened all titles and abstracts for eligibility. We read the full text of articles potentially eligible for inclusion and independently extracted data on study design, demographics and outcomes. Disagreements were resolved by collegial discussion. We included the following outcomes.

(a) ICH volume: mean ICH volume in millilitre $(\mathrm{ml})$ with standard deviation (SD); if median and interquartile range were provided, we extrapolated the mean and SD using a published formula and method [19].

(b) Haematoma expansion (HE), usually defined as haematoma volume increase of $+6 \mathrm{ml}$ or $+33 \%$ between baseline and follow-up imaging.

(c) Mortality: either in-hospital or 3-month mortality.

VKA was defined as the use of warfarin, phenprocoumon, fluindione or acénocoumarol. NOAC was defined as the use of apixaban, dabigatran, edoxaban or rivaroxaban.

\section{Risk of bias}

Two authors (DJS and MBG) independently assessed study quality and risk of bias using the scheme suggested by the Cochrane collaboration ("Tool to assess risk of bias in cohort studies").

\section{Statistical analysis}

We used Review Manager (RevMan) Version 5.3 (Copenhagen: The Nordic Cochrane Centre, The Cochrane Collaboration, 2014) statistical program package. Patients with VKA-ICH and NOAC-ICH were analysed separately and compared with patients with non-OAC ICH.

We used the Mantel-Haenszel random-effects method to compare mortality and $\mathrm{HE}$ and calculated odds ratios (OR) and corresponding 95\% confidence intervals (CI). $\mathrm{ICH}$ volumes as continuous variable were pooled using the difference of means comparison based on the mean ICH volume reported in each study and the corresponding SD. We reported the mean difference of ICH volume (in $\mathrm{ml}$ ) 
with corresponding SD. Heterogeneity was assessed using $\mathrm{I}^{2}$ statistics and displayed the results using forest plots.

\section{Results}

The literature research identified 226 publications. After reading the titles and abstracts/full papers if applicable, we included 19 studies (see Table 1): 12 publications from observational single-centre studies $[4,5,7-11,20-24]$ (all tertiary care centres-two publications from the same centre reporting complementary outcome data [7, 8]); six observational multi-centre studies [6, 12, 25-28] [one population-based study with two publications $[6,12]$ reporting complementary outcome data and one national US Get-with-the-guidelines (GWTG) registry [25]]; and one sub-analysis of the placebo arm of a randomized controlled trial [29]. If there were multiple publications from the same study/centre, this study/centre only contributed data from one publication to each outcome. Altogether, the studies reported data of 150,270 patients with ICH of whom 16,546 (11.0\%) had VKA-ICH, 4943 (3.3\%) had NOAC-ICH and $128,761(85.7 \%)$ had non-OAC ICH. The large US GWTG study [25] contributed 141,311 (94.0\%) patients but reported only outcome data for in-hospital mortality and not for haematoma volume, HE or 3-month mortality. The risk of bias for included studies was medium (Table 2).

\section{VKA-ICH vs. non-OAC ICH}

\section{Overview}

All 19 studies reported data of 16,546 patients with VKAICH. The number of patients with VKA-ICH ranged between 21 and 208 patients in single- and multi-centre studies, with the GWTG registry reporting data of 15,036 patients with VKA-ICH. Fourteen of 19 studies recruited less than 100 patients with VKA-ICH. Patients with VKAICH were significantly older than patients with non-OAC ICH (mean age difference: 5.55 years, 95\% CI 4.03-7.07, $p<0.0001, I^{2}=92 \%, p<0.001$, supplemental figure).

\section{Haematoma volume}

Fourteen studies [4, 7, 9-12, 20-24, 27-29] reported data on ICH volume in 981 patients with VKA-ICH compared to 4583 patients with non-OAC ICH. In 7 studies, haematoma volume was measured using the $\mathrm{ABC} / 2$ formula $[9,11,12$, 20,24], three studies used a planimetric software (ALICE or Analyze 10.0) [7, 21, 29]. Specifications of the used imaging techniques were provided in four studies [9-12, 24]. Details on applied methods considering ICH volume measurement are provided in the supplementary table. The mean ICH volume ranged from $19.9 \mathrm{ml}$ to $44.8 \mathrm{ml}$ in patients with VKA-ICH compared to $13.1 \mathrm{ml}$ to $44.6 \mathrm{ml}$ in non-OAC ICH. VKA-ICH was associated with significantly larger haematoma volume with a pooled mean volume difference of $9.66 \mathrm{ml}\left(95 \% \mathrm{CI} 6.24-13.07 \mathrm{ml}, p<0.00001 ; I^{2}=42 \%\right.$, $p=0.05$; Fig. 1).

\section{Haematoma expansion}

Eight studies [7, 9, 10, 20, 22, 24, 28, 29] reported data on HE of 302 patients with VKA-ICH compared to 1944 patients with non-OAC ICH. Overall, VKA-ICH was associated with a significantly increased risk of HE (OR 2.96, 95\% CI 1.74-4.97, $p<0.00001 ; I^{2}=65 \%, p=0.005$, Fig. 2$)$ with a pooled rate of $35.8 \%$ compared to $18.9 \%$ in patients with non-OAC ICH.

\section{Mortality}

Fifteen studies reported mortality: eight studies [4, 6, $21-23,25,26,28]$ reported in-hospital mortality in 15,803 VKA-ICH patients compared to 125,434 non-OAC ICH patients (of whom the majority were from the GWTG registry: 15,036 patients with VKA-ICH and 121,357 patients with non-OAC ICH). Eight studies [5, 8-10, 20, 23, 28, 29] reported 3-month mortality in 537 patients with VKAICH compared to 2951 patients with non-OAC ICH. VKAICH was associated with increased in-hospital mortality (Fig. 3; VKA-ICH: $32.8 \%$ vs. non-OAC ICH: $22.4 \%$; OR $1.83,95 \%$ CI $1.61-2.07, p<0.00001, I^{2}=20 \%, p=0.27$ ) and 3-month mortality (Fig. 4; VKA-ICH: $47.1 \%$ vs. nonOAC ICH: $25.5 \%$; OR 2.24, 95\%CI 1.52-3.31, $p<0.00001)$ although the data showed significant heterogeneity $\left(I^{2}=71 \%\right.$, $p=0.001$ ) and more recent studies (i.e. published since 2009 $[9,20,28])$ and those published 2017 [10, 23] from specialized stroke centres showed lower mortality rates than older studies [5, 8, 29].

\section{NOAC-ICH vs. non-OAC ICH}

Only 2 studies included patients with NOAC-ICH: one single-centre study [10] from a German tertiary hospital (25 NOAC-ICH patients) and the GWTG registry [25]. The latter reported only data on in-hospital mortality; thus there were insufficient studies to perform a meta-analysis. 


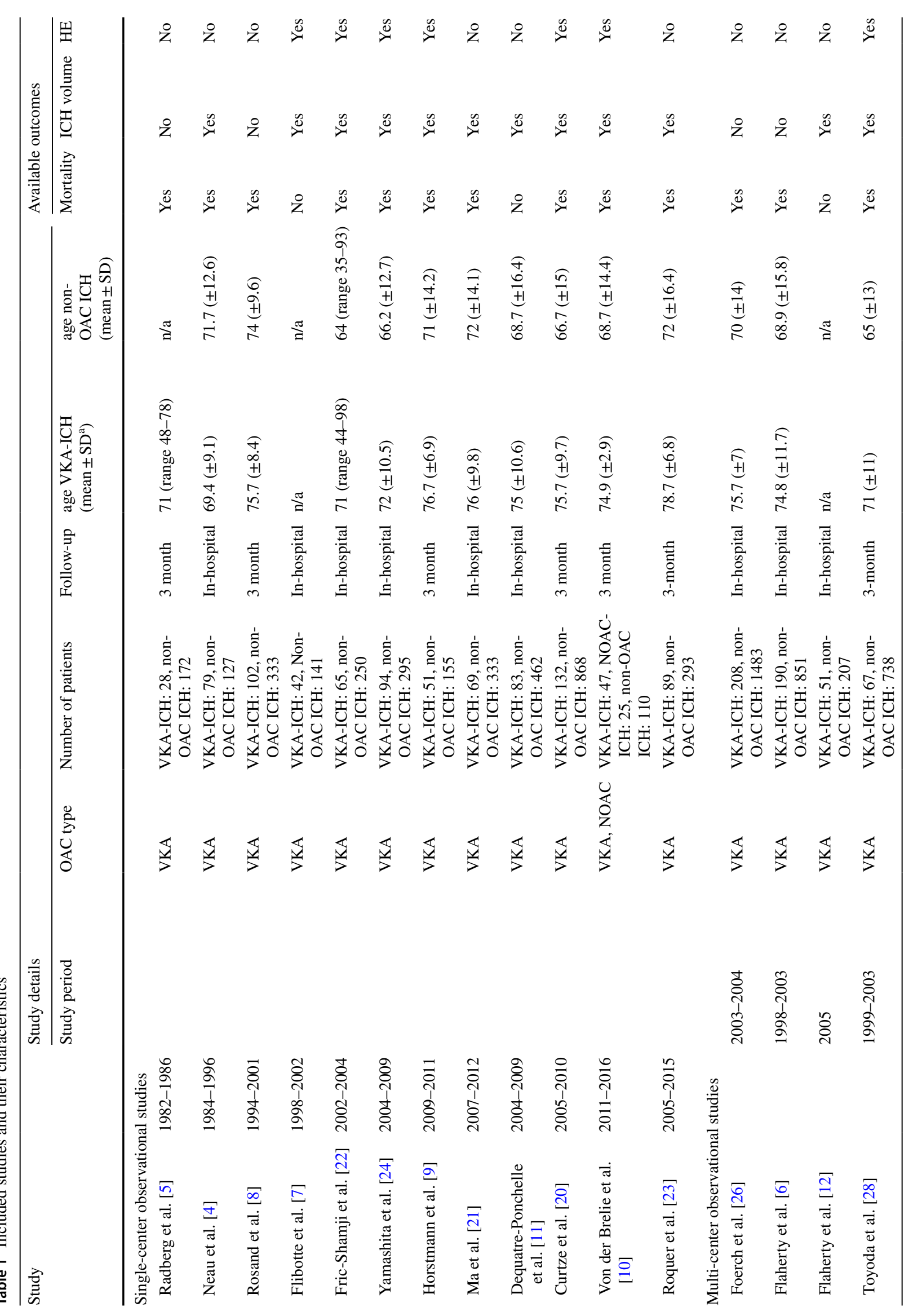




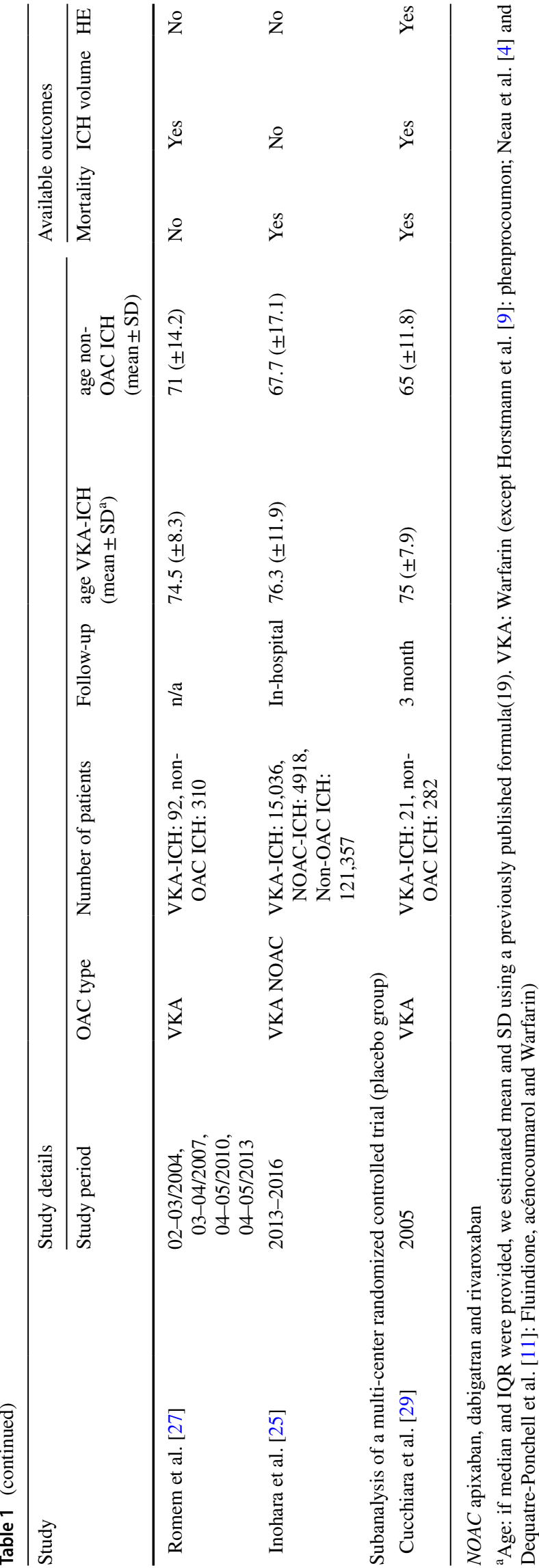

\section{Discussion}

This systematic review and meta-analysis provided the most precise available estimates on key prognostic factors and outcomes for OAC-ICH. Our main findings were that compared to non-OAC ICH, VKA-ICH is associated with: (1) a higher mean haematoma volume of about $10 \mathrm{ml}$; (2) nearly twice the risk of in-hospital and 3-month mortality; and (3) nearly triple the risk of HE. There are insufficient data to draw firm conclusions about NOAC-ICH compared to non-OAC ICH.

This meta-analysis confirms, refines and expands current knowledge on the effect of OAC on ICH derived from prior studies. Several studies $[4,5,12]$ described larger haematoma volumes in patients with VKA-ICH while more recent studies found no differences $[9,10]$ or differences only in subgroups of patients $[11,20]$. Our meta-analysis has clarified and expanded these findings. We found that VKA-ICH is associated with significantly larger haematoma volumes. The increase of nearly $10 \mathrm{ml}$ is clinically significant as haematoma volume is one of the most important predictors of poor outcome [30]; indeed, a haematoma volume of $\geq 30 \mathrm{ml}$ has been included in the ICH-score [31] to predict outcome in ICH.

HE is also an important predictor of poor outcome and potential target for treatment $[32,33]$. In the presence of $\mathrm{OAC}$, it is feared that continuous bleeding leads to more and significant $\mathrm{HE}$ and rapid reversal of the anticoagulant effect is a widely accepted treatment priority [34-38]. The first study [7] reporting increased HE rates found a large difference between VKA-ICH (54\%) and non-OAC ICH (16\%). Our meta-analysis confirmed a significantly higher rate of HE but our more precise estimate suggests that the difference is much smaller than initially reported, with HE in $36 \%$ of patients with VKA-ICH and $19 \%$ in patients with non-OAC ICH. Nevertheless, we found heterogeneity among studies included in our meta-analysis, notably differences between studies from North America $[7,22,29]$ reporting data from the early 2000 s and studies from Europe and Asia [9, 10, 20, 24, 28] reporting more recent data. Regional differences in anticoagulation reversal strategies although not systematically reported in all studies might have played a role. European centres tend to use PCC combined with FFP and Vitamin K as first-line therapy for VKA reversal $[35,36,38,39]$ while in the US, FFP combined with Vitamin $\mathrm{K}$ has been the mainstay [7, $36,37]$. A recent randomized controlled trial found that PCC is superior to FFP in rapidly reversing VKA-related clotting abnormalities, but this study was underpowered to find differences in clinical outcomes [40]. The HE rate of $36 \%$ in patients with VKA-ICH is in line with the results from studies comparing HE between VKA-ICH and 
Table 2 Risk of bias assessment according to the Cochrane 'Tool to Assess Risk of Bias in Cohort Studies"

\begin{tabular}{|c|c|c|c|c|c|c|c|c|}
\hline & 1 & 2 & 3 & 4 & 5 & 6 & 7 & 8 \\
\hline Radberg et al. [5] & ++ & + & + & -- & ++ & ++ & - & ++ \\
\hline Neau et al. [4] & + & ++ & + & - & - & ++ & + & + \\
\hline Rosand et al. [8] & ++ & ++ & ++ & ++ & ++ & ++ & ++ & + \\
\hline Flibotte et al. [7] & ++ & ++ & ++ & ++ & ++ & ++ & ++ & + \\
\hline Flaherty et al. [6] & + & + & ++ & ++ & ++ & ++ & + & + \\
\hline Flaherty et al. [12] & + & ++ & + & - & + & + & - & + \\
\hline Cucchiara et al. [29] & + & ++ & ++ & ++ & ++ & ++ & - & + \\
\hline Horstmann et al. [9] & ++ & + & + & + & + & ++ & + & + \\
\hline Ma et al. [21] & ++ & ++ & ++ & ++ & ++ & + & -- & + \\
\hline Dequatre-Ponchelle et al. [11] & ++ & ++ & ++ & - & + & ++ & -- & + \\
\hline Curtze et al. [20] & ++ & ++ & ++ & + & + & ++ & ++ & + \\
\hline Von der Brelie et al. [10] & ++ & + & ++ & + & + & ++ & ++ & ++ \\
\hline Inohara et al. [25] & ++ & ++ & ++ & ++ & + & ++ & + & + \\
\hline Romem [27] & ++ & ++ & ++ & ++ & + & + & + & + \\
\hline Roquer [23] & ++ & - & - & + & + & + & - & + \\
\hline Toyoda [28] & + & ++ & ++ & ++ & + & ++ & + & + \\
\hline Fric-Shamji [22] & ++ & + & ++ & + & + & ++ & + & + \\
\hline Foerch [26] & ++ & + & ++ & + & + & + & -- & + \\
\hline Yamashita et al. [24] & ++ & ++ & ++ & + & - & + & + & + \\
\hline
\end{tabular}

1. Was selection of exposed and non-exposed cohorts drawn from the same population?

2. Can we be confident in the assessment of exposure?

3. Can we be confident that the outcome of interest was not present at start of study?

4. Did the study match exposed and unexposed for all variables that are associated with the outcome of interest or did the statistical analysis adjust for these prognostic variables?

5. Can we be confident in the assessment of the presence or absence of prognostic factors?

6. Can we be confident in the assessment of outcome?

7. Was the follow-up of cohorts adequate?

8. Were co-interventions similar between groups?

Ratings: ++ , definitely yes (Low risk of bias); + , probably yes; -, probably no; --, definitely no (high risk of bias)

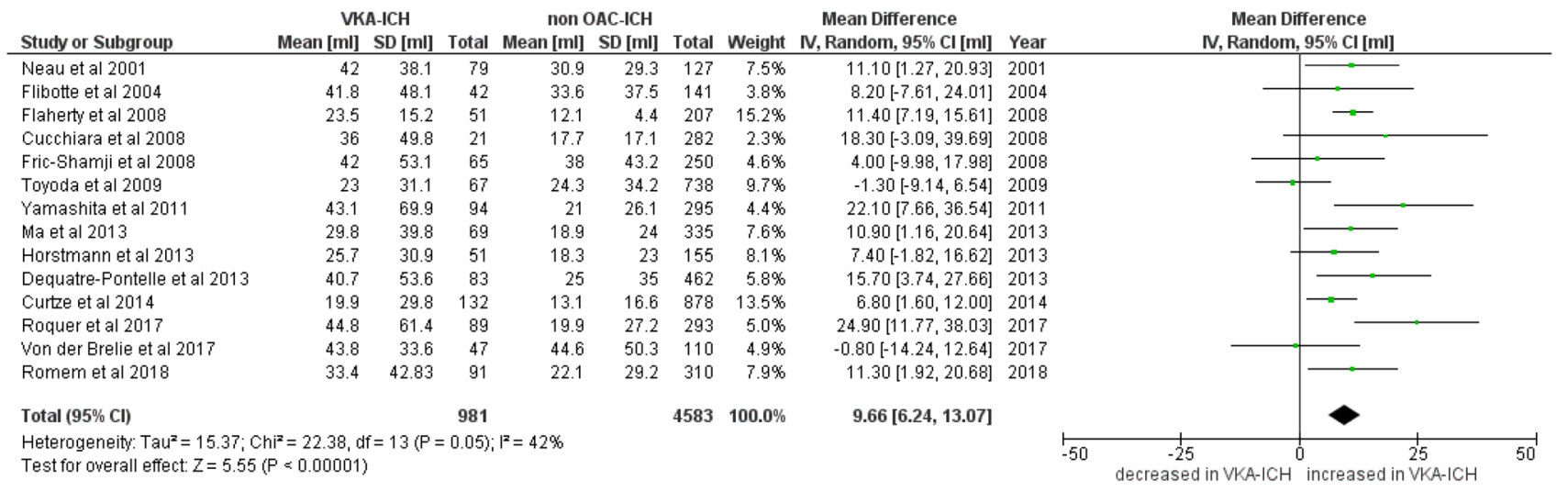

Fig. 1 Mean difference of ICH volume in VKA-ICH compared to non-OAC ICH 


\begin{tabular}{|c|c|c|c|c|c|c|c|c|c|c|c|c|}
\hline \multirow[b]{2}{*}{ Study or Subgroup } & \multicolumn{2}{|c|}{ VKA-ICH } & \multicolumn{2}{|c|}{ non-OAC ICH } & \multicolumn{3}{|c|}{ Odds Ratio } & \multirow{2}{*}{\multicolumn{5}{|c|}{$\begin{array}{c}\text { Odds Ratio } \\
\text { M-H, Random, } 95 \% \mathrm{Cl}\end{array}$}} \\
\hline & Events & Total & Events & Total & Weight & M-H, Random, $95 \% \mathrm{Cl}$ & Year & & & & & \\
\hline Flibotte et al 2004 & 7 & 13 & 9 & 57 & $9.0 \%$ & $6.22[1.69,22.88]$ & 2004 & & & & & $\longrightarrow$ \\
\hline Cucchiara et al 2008 & 10 & 18 & 69 & 267 & $12.1 \%$ & $3.59[1.36,9.45]$ & 2008 & & & & & \\
\hline Fric-Shamji et al 2008 & 34 & 65 & 31 & 215 & $16.1 \%$ & $6.51[3.51,12.08]$ & 2008 & & & & & + \\
\hline Toyoda et al 2009 & 21 & 54 & 120 & 612 & $16.6 \%$ & $2.61[1.46,4.67]$ & 2009 & & & & & \\
\hline Yamashita et al 2011 & 6 & 54 & 27 & 213 & $12.4 \%$ & $0.86[0.34,2.20]$ & 2011 & & & & & \\
\hline Horstmann et al 2013 & 4 & 12 & 14 & 120 & $8.9 \%$ & $3.79[1.01,14.22]$ & 2013 & & & & & \\
\hline Curtre et al 2014 & 21 & 63 & 94 & 383 & $16.7 \%$ & $1.54[0.87,2.73]$ & 2014 & & & & & \\
\hline Von der Brelie et al 2017 & 5 & 23 & 4 & 77 & $8.2 \%$ & $5.07[1.23,20.81]$ & 2017 & & & & & \\
\hline Total $(95 \% \mathrm{Cl})$ & & 302 & & 1944 & $100.0 \%$ & $2.96[1.76,4.97]$ & & & & & & \\
\hline Total events & 108 & & 368 & & & & & & & & & \\
\hline $\begin{array}{l}\text { Heterogeneity: } \operatorname{Tau}^{2}=0.3 \\
\text { Test for overall effect: } Z=\end{array}$ & $\begin{array}{l}\mathrm{Chi}^{2}=20 \\
10(\mathrm{P}=0\end{array}$ & $\begin{array}{l}.10, \mathrm{df} \\
.0001)\end{array}$ & $=7(\mathrm{P}=0$ & $005) ; I^{2}$ & $=65 \%$ & & & 0.1 & $\begin{array}{l}0.2 \\
\text { decre }\end{array}$ & $\begin{array}{ll}0.5 \\
\text { in } \mathrm{V} / \mathrm{KA}-\mathrm{ICH}\end{array}$ & increased & $\begin{array}{c}5 \\
\mathrm{CH}\end{array}$ \\
\hline
\end{tabular}

Fig. 2 Rate of haematoma expansion (HE) in VKA-ICH compared to non-OAC ICH

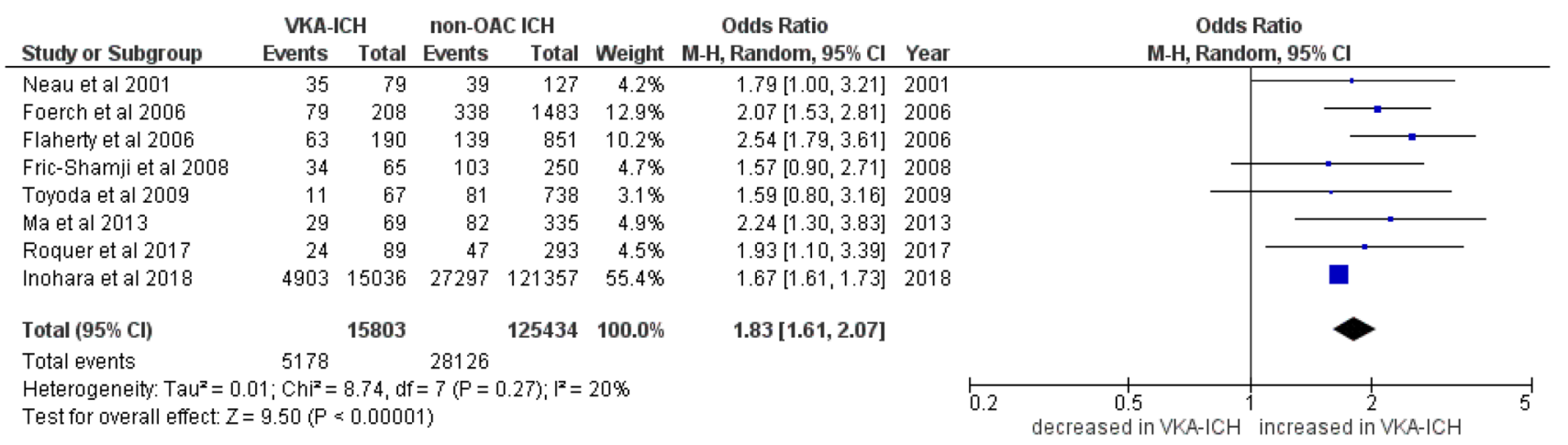

Fig. 3 In-hospital mortality in VKA-ICH compared to non-OAC ICH

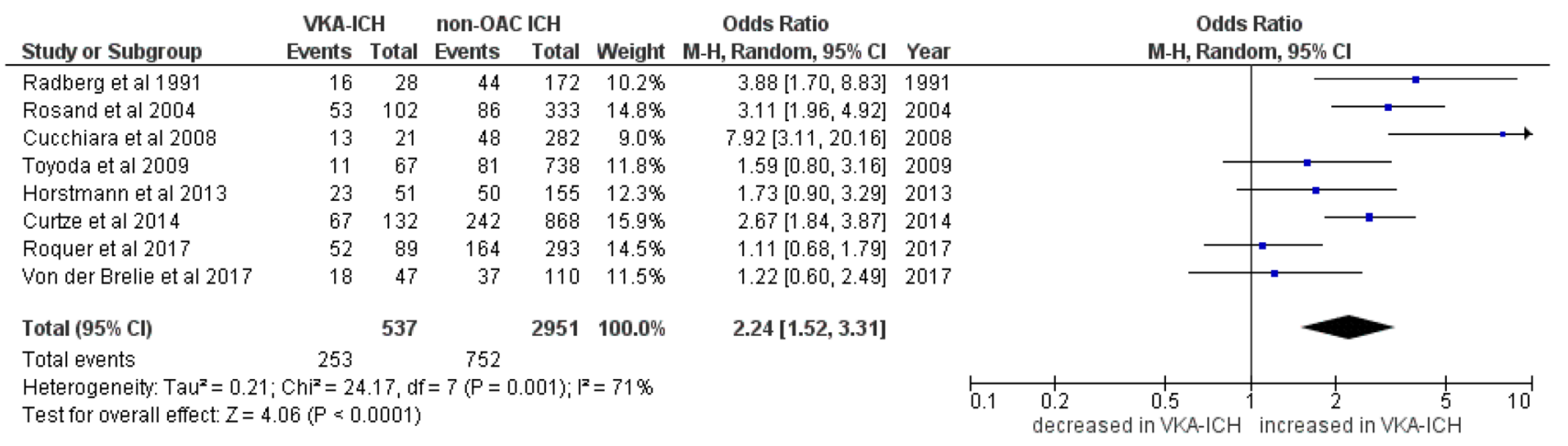

Fig. 4 3-month mortality in VKA-ICH compared to non-OAC ICH

NOAC-ICH, which reported HE rates between 34\% [16] and $37 \%$ [17] for patients with VKA-ICH. Another study investigating treatment strategies for VKA-ICH reported an overall HE rate of $36 \%$ in VKA-ICH [35]. Interestingly, the latter study found a HE rate of only $18 \%$ in VKA-ICH patients receiving most aggressive treatment (INR $<1.3$ within $1 \mathrm{~h}$ and systolic blood pressure $<160 \mathrm{mmHg}$ within $4 \mathrm{~h}$ ) which is about the same rate as we found in non-OAC
ICH (19\%). This might point towards important treatment effects in VKA-ICH, suggesting potential normalization of HE rates if VKA-ICH is treated aggressively.

Although we found increased mortality in patients with VKA-ICH confirming prior research $[4,5,8]$, there was significant heterogeneity among the published studies. Studies reporting data from the early 2000s or 1990s had worse outcome while more recent studies had less-pronounced 
differences. This might in part be explained by recent developments in acute blood pressure treatment (following INTERACT-2 [41] published in 2013) and widespread introduction of stroke unit care for patients with ICH [42]. Patients with VKA-ICH may particularly benefit from rapid and aggressive therapies in multi-disciplinary dedicated stroke unit teams. An aforementioned study [35] investigating treatment effects in VKA-ICH found the overall inhospital mortality in VKA-ICH to be $31 \%$ which is in line with the findings of our meta-analysis (32.8\%). Furthermore, in this study, patients receiving rapid blood pressure control and anticoagulation reversal had in-hospital mortality as low as only $13 \%$, even lower as the $22 \%$ that we found in the non-OAC ICH group of our meta-analysis. Taken together, although our meta-analysis found HE and mortality to be higher in VKA-ICH, the heterogeneity of data included in our meta-analysis together with data from other studies might indicate that intensive treatment can significantly mitigate excessive HE and mortality reducing it to a level comparable to that of non-OAC ICH.

While many studies investigated differences between VKA-ICH and NOAC-ICH [13-17], we identified only 2 studies comparing NOAC-ICH with non-OAC ICH. Data from the US GWTG registry [25] found an increased inhospital mortality but did not report data on haematoma volume, HE or 3-month mortality. The study from Von der Brelie et al. [10] found increased haematoma volume, $\mathrm{HE}$ and 3 -month mortality in NOAC-ICH but this study included only 25 patients with NOAC-ICH.

Our study has several strengths: 1) combining and pooling data from multiple, independent studies with a large number of patients with VKA-ICH, we overcome limitations from prior smaller studies, refining and expanding their findings; 2) combining data from older with more recent studies allowed to highlight possible improvements in treatment of VKA-ICH underlining that further efforts to improve treatment are still important; 3) independent literature research and extraction of study data from two investigators reduced bias and increased data validity.

Our study has the following limitations: (1) we used aggregate observational data which are always prone to bias; (2) we were not able to perform multivariate analysis accounting for other potential predictors of haematoma volume, HE and mortality including age (VKA-ICH patients were 5 years older), acute treatment (blood pressure control and anticoagulation reversal) and other factors (e.g. comorbidities); (3) we were not able to calculate gender- or age-specific results. Furthermore, we did not have access to INR results at presentation. (4) Different methods applied to assess haematoma volumes have certainly contributed to the observed heterogeneity of results and are a source of bias. However, these methodological limitations applies to both, OAC-ICH and non-OAC-ICH. (5) Even with sophisticated measuring techniques, for large haematomas, the magnitude of haematoma volume measurement error may exceed the threshold used to dichotomize HE in the respective study [43]. In our analysis of aggregate data, we were not able to correct for this incertitude.

To summarize, this meta-analysis provided precise estimates of key prognostic variables and outcomes: we showed that VKA-ICH has larger haematoma volume, more frequent HE and higher mortality than non-VKAICH. Studies comparing NOAC-ICH and non-OAC ICH are scarce and current knowledge on NOAC-ICH is only available in relation to the effect of VKA-ICH. Further research on the effect of NOAC on these outcomes is needed.

Acknowledgements DJS is supported by a fellowship from the Swiss National Science Foundation (SNF), the Bangerter-Rhyner Foundation, the Bayer Foundation 2017 "Thrombosis Research Award" and the Swiss Society of Neurology. MBG has received a Young Talents in Clinical Research grant by the Swiss Academy of Medical Sciences and the Bangerter-Rhyner-Foundation. UF was supported from the Swiss National Science Foundation (SNF 32003B_169975) and the Swiss Heart Foundation. DJW receives research funding from the British Heart Foundation and The Stroke Association and is supported by the National Institute for Health Research University College London Hospitals Biomedical Research Centre.

Author contribution DJS and DJW conceived the study and wrote the first draft of the manuscript. DJS and MBG collected data and performed the statistical analysis. All authors contributed to collection and interpretation of the data and editing the manuscript for important intellectual content.

Funding DJS was supported by research grants from the Swiss National Science Foundation, the Bangerter-Rhyner Foundation, the Swiss Society of Neurology and the 2017 Bayer Foundation Thrombosis Award. MBG has received a "Young Talents in Clinical Research" grant by the Swiss Academy of Medical Sciences and the BangerterRhyner-Foundation (YTCR grant 13_18)UF was supported from the Swiss National Science Foundation (SNF 32003B_169975) and the Swiss Heart Foundation.

\section{Compliance with ethical standards}

Conflicts of interest DJS: scientific advisory boards: Bayer and Pfizer. Compensation for educational efforts: Stago. MBG: no disclosures. TT: academic grants from Helsinki University Central Hospital, University of Gothenburg, Sahlgrenska University Hospital, and Sigrid Juselius Foundation for research on intracerebral hemorrhages. TT has or has had research contracts with Boehringer-Ingelheim, Bayer, Pfizer, and Portola Pharm. Advisory board membership for BoehringerIngelheim, Bayer, Pfizer, and Lumosa Pharm. PAL: scientific advisory boards: Bayer, Daiichi-Sankyo and Boehringer Ingelheim. Funding for travel or speaker honoraria: Bayer and Boehringer Ingelheim. Research funding: Boehringer Ingelheim. UF: consultant for Medtronic and Stryker, a Co-PI of the SWITCH trial and the ELAN trial (both supported by the Swiss National Science Foundation and the Swiss Heart Foundation) and of the SWIFT DIRECT trial (supported by Medtronic). Speaker honoraria payed to the institution by AMGEN. STE: received funding for travel or speaker honoraria from Bayer, Boehringer Ingelheim and Daiichi-Sankyo. He has served on scientific 
advisory boards for Bayer, Boehringer Ingelheim, BMS/Pfizer, and MindMaze and on the editorial board of Stroke. His institutions have received an educational grant from Pfizer, compensation from Stago for educational efforts and research support from Daiichi-Sankyo, the Science Funds [Wissenschaftsfonds] of the University Hospital Basel, the University Basel, the "Freiwillige Akademische Gesellschaft Basel", the Swiss Heart Foundation, and the Swiss National Science Foundation. DJW: speaker honoraria from Bayer AG. MP reports honoraria as a member of the speaker bureau of Aspen, Sanofi-Aventis, Boehringer Ingelheim, Bayer AG, Bristol-Myers Squibb, Medtronic, and Pfizer.

Ethical standard This aricle does not contain any studies with human participants or anmals performed by any of the authors.

Open Access This article is distributed under the terms of the Creative Commons Attribution 4.0 International License (http://creativeco mmons.org/licenses/by/4.0/), which permits unrestricted use, distribution, and reproduction in any medium, provided you give appropriate credit to the original author(s) and the source, provide a link to the Creative Commons license, and indicate if changes were made.

\section{References}

1. Poon MT, Fonville AF, Al-Shahi Salman R (2014) Longterm prognosis after intracerebral haemorrhage: systematic review and meta-analysis. J Neurol Neurosurg Psychiatry 85(6):660-667

2. Salazar CA, del Aguila D, Cordova EG (2014) Direct thrombin inhibitors versus vitamin $\mathrm{K}$ antagonists for preventing cerebral or systemic embolism in people with non-valvular atrial fibrillation. Cochrane Database Syst Rev. https://doi. org/10.1002/14651858.CD009893.pub2

3. Bruins KM, Slot KM, Berge E (2018) Factor Xa inhibitors versus vitamin $\mathrm{K}$ antagonists for preventing cerebral or systemic embolism in patients with atrial fibrillation. Cochrane Database Syst Rev. https://doi.org/10.1002/14651858.CD008980.pub3

4. Neau JP, Couderq C, Ingrand P, Blanchon P, Gil R (2001) Intracranial hemorrhage and oral anticoagulant treatment. Cerebrovasc Dis (Basel, Switzerland) 11(3):195-200

5. Radberg JA, Olsson JE, Radberg CT (1991) Prognostic parameters in spontaneous intracerebral hematomas with special reference to anticoagulant treatment. Stroke 22(5):571-576

6. Flaherty ML, Haverbusch M, Sekar P, Kissela BM, Kleindorfer D, Moomaw CJ et al (2006) Location and outcome of anticoagulantassociated intracerebral hemorrhage. Neurocrit Care 5(3):197-201

7. Flibotte JJ, Hagan N, O'Donnell J, Greenberg SM, Rosand J (2004) Warfarin, hematoma expansion, and outcome of intracerebral hemorrhage. Neurology 63(6):1059-1064

8. Rosand J, Eckman MH, Knudsen KA, Singer DE, Greenberg SM (2004) The effect of warfarin and intensity of anticoagulation on outcome of intracerebral hemorrhage. Arch Intern Med 164(8):880-884

9. Horstmann S, Rizos T, Lauseker M, Mohlenbruch M, Jenetzky E, Hacke W et al (2013) Intracerebral hemorrhage during anticoagulation with vitamin K antagonists: a consecutive observational study. J Neurol. https://doi.org/10.1007/s00415-013-6939-6

10. von der Brelie C, Doukas A, Naumann R, Dempfle A, Larsen $\mathrm{N}$, Synowitz M et al (2017) Clinical and radiological course of intracerebral haemorrhage associated with the new non-vitamin K anticoagulants. Acta Neurochir 159(1):101-109
11. Dequatre-Ponchelle N, Henon H, Pasquini M, Rutgers MP, Bordet R, Leys D et al (2013) Vitamin K antagonists-associated cerebral hemorrhages: what are their characteristics? Stroke 44(2):350-355

12. Flaherty ML, Tao H, Haverbusch M, Sekar P, Kleindorfer D, Kissela B et al (2008) Warfarin use leads to larger intracerebral hematomas. Neurology 71(14):1084-1089

13. Lioutas VA, Goyal N, Katsanos AH, Krogias C, Zand R, Sharma VK et al (2018) Clinical outcomes and neuroimaging profiles in nondisabled patients with anticoagulant-related intracerebral hemorrhage. Stroke 49(10):2309-2316

14. Wilson D, Charidimou A, Shakeshaft C, Ambler G, White $\mathrm{M}$, Cohen $\mathrm{H}$ et al (2016) Volume and functional outcome of intracerebral hemorrhage according to oral anticoagulant type. Neurology 86(4):360-366

15. Tsivgoulis G, Wilson D, Katsanos AH, Sargento-Freitas J, Marques-Matos C, Azevedo E et al (2018Neuroimaging) Neuroimaging and clinical outcomes of oral anticoagulant-associated intracerebral hemorrhage. Ann Neurol. https://doi.org/10.1002/ ana. 25342

16. Wilson D, Seiffge DJ, Traenka C, Basir G, Purrucker JC, Rizos $\mathrm{T}$ et al (2017) Outcome of intracerebral hemorrhage associated with different oral anticoagulants. Neurology 88(18):1693-1700

17. Tsivgoulis G, Lioutas VA, Varelas P, Katsanos AH, Goyal N, Mikulik R et al (2017) Direct oral anticoagulant- vs vitamin $\mathrm{K}$ antagonist-related nontraumatic intracerebral hemorrhage. Neurology. https://doi.org/10.1212/WNL.0000000000004362

18. Liberati A, Altman DG, Tetzlaff J, Mulrow C, Gotzsche PC, Ioannidis JP et al (2009) The PRISMA statement for reporting systematic reviews and meta-analyses of studies that evaluate healthcare interventions: explanation and elaboration. BMJ (Clin Res ed) 339:b2700

19. Wan X, Wang W, Liu J, Tong T (2014) Estimating the sample mean and standard deviation from the sample size, median, range and/or interquartile range. BMC Med Res Methodol $14: 135$

20. Curtze S, Strbian D, Meretoja A, Putaala J, Eriksson H, Haapaniemi E et al (2014) Higher baseline international normalized ratio value correlates with higher mortality in intracerebral hemorrhage during warfarin use. Eur J Neurol 21(4):616-622

21. Ma M, Meretoja A, Churilov L, Sharma GJ, Christensen S, Liu $X$ et al (2013) Warfarin-associated intracerebral hemorrhage: volume, anticoagulation intensity and location. J Neurol Sci 332(1-2):75-79

22. Fric-Shamji EC, Shamji MF, Cole J, Benoit BG (2008) Modifiable risk factors for intracerebral hemorrhage: study of anticoagulated patients. Can Fam Physician 54(8):1138-1139

23. Roquer J, Vivanco Hidalgo RM, Ois A, Rodriguez Campello A, Cuadrado Godia E, Giralt Steinhauer E et al (2017) Antithrombotic pretreatment increases very-early mortality in primary intracerebral hemorrhage. Neurology 88(9):885-891

24. Yamashita S, Kimura K, Iguchi Y, Shibazaki K (2011) Prior oral antithrombotic therapy is associated with early death in patients with supratentorial intracerebral hemorrhage. Internal Med (Tokyo, Japan) 50(5):413-419

25. Inohara T, Xian Y, Liang L, Matsouaka RA, Saver JL, Smith EE et al (2018) Association of intracerebral hemorrhage among patients taking non-vitamin $\mathrm{K}$ antagonist vs vitamin $\mathrm{K}$ antagonist oral anticoagulants with in-hospital mortality. JAMA, J Am Med Assoc 319(5):463-473

26. Foerch C, Sitzer M, Steinmetz H, Neumann-Haefelin T (2006) Pretreatment with antiplatelet agents is not independently associated with unfavorable outcome in intracerebral hemorrhage. Stroke 37(8):2165-2167

27. Romem R, Tanne D, Geva D, Einhorn-Cohen M, Shlomo N, Bar-Yehuda $S$ et al (2018) Antithrombotic treatment prior to 
intracerebral hemorrhage: analysis in the national acute stroke israeli registry. J Stroke Cerebrovasc Dis 27(11):3380-3386

28. Toyoda K, Yasaka M, Nagata K, Nagao T, Gotoh J, Sakamoto T et al (2009) Antithrombotic therapy influences location, enlargement, and mortality from intracerebral hemorrhage. The Bleeding with Antithrombotic Therapy (BAT) Retrospective Study. Cerebrovasc Dis 27(2):151-159

29. Cucchiara B, Messe S, Sansing L, Kasner S, Lyden P (2008) Hematoma growth in oral anticoagulant related intracerebral hemorrhage. Stroke 39(11):2993-2996

30. Broderick JP, Brott TG, Duldner JE, Tomsick T, Huster G (1993) Volume of intracerebral hemorrhage. A powerful and easy-to-use predictor of 30-day mortality. Stroke 24:987-993

31. Hemphill JC 3rd, Bonovich DC, Besmertis L, Manley GT, Johnston SC (2001) The ICH score: a simple, reliable grading scale for intracerebral hemorrhage. Stroke 32(4):891-897

32. Demchuk AM, Dowlatshahi D, Rodriguez-Luna D, Molina CA, Blas YS, Dzialowski I et al (2012) Prediction of haematoma growth and outcome in patients with intracerebral haemorrhage using the CT-angiography spot sign (PREDICT): a prospective observational study. Lancet Neurol 11(4):307-314

33. Dowlatshahi D, Demchuk AM, Flaherty ML, Ali M, Lyden PL, Smith EE (2011) Defining hematoma expansion in intracerebral hemorrhage: relationship with patient outcomes. Neurology 76(14):1238-1244

34. Dowlatshahi D, Butcher KS, Asdaghi N, Nahirniak S, Bernbaum ML, Giulivi A et al (2012) Poor prognosis in warfarin-associated intracranial hemorrhage despite anticoagulation reversal. Stroke 43(7):1812-1817

35. Kuramatsu JB, Gerner ST, Schellinger PD, Glahn J, Endres M, Sobesky J et al (2015) Anticoagulant reversal, blood pressure levels, and anticoagulant resumption in patients with anticoagulation-related intracerebral hemorrhage. JAMA J Am Med Assoc 313(8):824-836
36. Parry-Jones AR, Di Napoli M, Goldstein JN, Schreuder FH, Tetri S, Tatlisumak T et al (2015) Reversal strategies for vitamin $\mathrm{K}$ antagonists in acute intracerebral hemorrhage. Ann Neurol 78(1):54-62

37. Hemphill JC 3rd, Greenberg SM, Anderson CS, Becker K, Bendok BR, Cushman M et al (2015) Guidelines for the management of spontaneous intracerebral hemorrhage: a guideline for healthcare professionals From the American Heart Association/American Stroke Association. Stroke 46(7):2032-2060

38. Steiner T, Al-Shahi Salman R, Beer R, Christensen H, Cordonnier C, Csiba L et al (2014) European Stroke Organisation (ESO) guidelines for the management of spontaneous intracerebral hemorrhage. Int J Stroke. https://doi.org/10.1111/ijs.12309

39. Rizos T, Jenetzky E, Herweh C, Hug A, Hacke W, Steiner T et al (2010) Point-of-care reversal treatment in phenprocoumon-related intracerebral hemorrhage. Ann Neurol 67(6):788-793

40. Steiner T, Poli S, Griebe M, Husing J, Hajda J, Freiberger A et al (2016) Fresh frozen plasma versus prothrombin complex concentrate in patients with intracranial haemorrhage related to vitamin $\mathrm{K}$ antagonists (INCH): a randomised trial. Lancet Neurol 15(6):566-573

41. Anderson CS, Heeley E, Huang Y, Wang J, Stapf C, Delcourt C et al (2013) Rapid blood-pressure lowering in patients with acute intracerebral hemorrhage. N Engl J Med 368(25):2355-2365

42. Langhorne P, Fearon P, Ronning OM, Kaste M, Palomaki H, Vemmos K et al (2013) Stroke unit care benefits patients with intracerebral hemorrhage: systematic review and meta-analysis. Stroke 44(11):3044-3049

43. Rodriguez-Luna D, Boyko M, Subramaniam S, Klourfeld E, Jo P, Diederichs BJ et al (2016) Magnitude of hematoma volume measurement error in intracerebral hemorrhage. Stroke 47(4):1124-1126

\section{Affiliations}

\section{David J. Seiffge ${ }^{1,2,3}$ (D) Martina B. Goeldlin ${ }^{3,4} \cdot$ Turgut Tatlisumak $^{5,6} \cdot$ Philippe Lyrer $^{2}$. Urs Fischer ${ }^{3}$. Stefan T. Engelter ${ }^{2,7} \cdot$ David J. Werring ${ }^{1}$}

1 Stroke Research Centre, Institute of Neurology, University College London, Russell Square House, 10 Russell Square, London, UK

2 Stroke Center and Neurology, Department of Clinical Research, University Hospital and University, Basel, Switzerland

3 Department of Neurology, Inselspital, Bern University Hospital, University of Bern, Bern, Switzerland

4 University Institute for Diagnostic and Interventional Neuroradiology, Inselspital, Bern University Hospital, University of Bern, Bern, Switzerland
5 Department of Clinical Neuroscience/Neurology, Institute of Neurosciences and Physiology, Sahlgrenska Academy at University of Gothenburg, Gothenburg, Sweden

6 Department of Neurology, Sahlgrenska University Hospital, Gothenburg, Sweden

7 Neurorehabilitation Unit, University Center for Medicine of Aging and Rehabilitation Basel, Felix Platter Hospital, University of Basel, Basel, Switzerland 